\title{
Contenido de ácidos grasos en carne de cuy
}

\section{Fatty acid content obtained from the guinea pig meat}

Fecha de recepción: 21 de diciembre de 2014

Fecha de aprobación: 08 de abril de 2015

\author{
César Iván Flores-Mancheno', Manuel Roca-Argüelles² \\ René Tejedor-Arias ${ }^{3}$ Iván Patricio Salgado-Tello ${ }^{4}$ \\ Nelson Ramiro Villegas-Soto ${ }^{5}$
}

\section{Resumen}

El objetivo del estudio fue determinar la composición de ácidos grasos en carne de cuy. El trabajo se desarrolló en la ciudad de Riobamba (Ecuador), y las líneas de cuyes utilizadas fueron tres: Criolla, Andina y Peruana mejorada. Se realizó análisis de varianza para las diferencias, comparación de medias según Duncan $(p<0.05)$. El contenido total de ácidos grasos saturados en la carne de este roedor no registró diferencias estadísticas entre las líneas estudiadas, ya que presentaron valores de 37,11, 37,01 y 36,71\%, para cuyes Criollo, Andino y Peruano mejorado, respectivamente; igualmente, el contenido de ácidos monoinsaturados tampoco registró diferencias estadísticas entre las tres líneas, pues se reportaron niveles de 30,49, 29,26 y 31,44\%, ni los niveles de ácidos grasos poliinsaturados, que fueron de 13,30, 11,04 y $14,22 \%$.

Palabras clave: Cuy, ácidos grasos en el cuy.

\begin{abstract}
The study's aim was to determine the fatty acid composition from the guinea pig meat. The study was conducted in the Riobamba City, Ecuador. The guinea pigs lines used were: Creole, Andean and Improved Peruvian. It was carried out a variance analysis for the differences and measurements comparison according Duncan $(\mathrm{P}<0.05)$. The total content of saturated fatty acids in this rodent meat did not registered statistical differences between the lines studied, with values of 37,11,37,01 and 36,71\% for the guinea pigs Creole, Andean and improved Peruvian respectively.

Neither the content of monounsaturated acids reported statistical differences between the three lines, as

1 M.Sc. Escuela Superior Politécnica de Chimborazo (Riobamba, Ecuador). c_flores@espoch.edu.ec.

2 Ph.D. Instituto de Investigaciones para la Industria Alimenticia - Universidad dē La Habana (La Habana, Cuba). mrocaa@ iiia.edu.cu.

3 Ph.D. Instituto de Farmacia y Alimentos - Universidad de La Habana (La Habana, Cuba). tejedor@ifal.uh.cu.

4 M.Sc. Profesional Independiente (Riobamba, Ecuador).

5 M.Sc. Profesional Independiente (Riobamba, Ecuador).
\end{abstract}


they showed levels of 30.49, 29.26 and 31.44\% respectively for Creole, Andean and Peruvian Improved lines, with no statistical differences.

Levels of polyunsaturated fatty acids in the meat of this rodent were 13,30, 11,04 and 14,22\% in Creole, Andean and Peruvian Improved, having no statistical difference between them.

Keywords: Guinea pigs, fatty acids. 


\section{Introducción}

La carne de cuy es magra, tiene un contenido bajo de grasa, alto en proteína y bajo en colesterol y sodio, que la hacen ideal para incluirla en una alimentación apta para todos los grupos poblacionales, desde niños hasta ancianos, y en diversas situaciones fisiológicas, como, por ejemplo, el embarazo o la etapa de lactancia (1). Esta carne es consumida principalmente como plato típico asado o en locro (guisada) en países como Perú, Ecuador, Colombia y Bolivia, ya que es muy sabrosa, suave, con alta calidad nutritiva y de fácil digestión (2), y ofrece grandes beneficios para la salud humana, ya que es fuente de proteína, hierro y vitamina $\mathrm{B}_{12}$; por esta razón la posicionan como un excelente alimento dietético (3). La carne de cuy tiene un contenido de grasa inferior al $15 \%$ y de proteína superior a $14 \%$ (4).

Los ácidos grasos en la carne pueden ser clasificados en saturados e insaturados, y estos últimos divididos en monoinsaturados y poliinsaturados, los cuales se subdividen en Omega $6(\omega 6)$ y Omega $3(\omega 3)$, y son considerados esenciales debido a la incapacidad del organismo humano de sintetizarlos, motivo por el cual deben ser incorporados en la dieta (5). Se considera, mediante referencias de estudios, que la carne es un producto que contiene $1 \mathrm{~g}$ de AGT/100 g (6). Al analizar los efectos del consumo de ácidos grasos trans (AGT) en la salud humana, se considera que son adversos, e incluso que causan mayor daño que el producido por las grasas saturadas; este efecto se ha visto reflejado principalmente en alteraciones del perfil lipídico, del sistema cardiovascular, relacionados con la diabetes mellitus, ciertos tipos del cáncer y la obesidad (7).

\section{Materiales y métodos}

Para el trabajo se seleccionaron animales adultos que pesaron entre 898,00 y $1460,00 \mathrm{~g}$; se utilizaron 15 unidades experimentales, distribuidas en tres líneas genéticas (Criollo, Andino y Peruano Mejorado), procedentes de la Unidad de Especies Menores de la Facultad de Ciencias Pecuarias de la $\mathrm{ESPOCH}$, que aplica un sistema de crianza técni- co, con alimentación a base de alfalfa y desechos de cosecha, complementada con concentrado; se tomaron en cuenta 5 repeticiones por línea genética, en donde cada unidad experimental está conformada por un animal. Una vez seleccionados los curíes se procedió al sacrificio, aplicando primero el aturdimiento, sacudiendo y estirando al roedor con relativa fuerza, y luego el degüello, desangrado, escaldado, pelado, lavado y eviscerado, eliminando cabeza y patas (8).

Los análisis experimentales se realizaron en el Laboratorio de Control y Análisis de los Alimentos (LACONAL), de la Facultad de Ciencias Alimentarias de la Universidad Técnica de Ambato (Ecuador). Se utilizó el método de cromatografía de gases para la determinación de los diferentes tipos de ácidos grasos: saturados, monoinsaturados, polinsaturados y transinsaturados (9).

Para los cálculos estadísticos se utilizó el software InfoStat versión libre (10) y el SPSS versión 21 (11). Los valores obtenidos de los experimentos se sometieron al análisis de varianza para las diferencias, comparación de medias según Duncan $(p<$ 0.05), desde el punto de vista determinación de ácidos grasos obtenidos de la carne de cuy.

\section{Resultados y discusión}

El contenido total de ácidos grasos saturados va a depender de ciertos factores, como la especie del animal y su alimentación; es así como carnes de especies como conejos, ovinos de engorde y bovinos $(12,13,14)$ presentan niveles superiores, mientras que en suinos (15) los valores son inferiores a los de la carne de cuy, que no registró diferencias estadísticas entre las tres líneas estudiadas, ya que presentaron valores de 37,11, 37,01 y 36,71\% en los animales Criollo, Andino y Peruano Mejorado, como se muestra en la Tabla I.

La Tabla I indica que en la carne de cobayos Peruano Mejorado, Andino y Criollo, los niveles de ácido láurico (C12:0) fueron 0,57; 0,57 y 0,56\%, respectivamente, no reportándose diferencias estadísticas entre las líneas estudiadas, con contenidos inferiores con respecto a conejos y ovinos $(12,13)$, y superiores al de los bovinos (14). 
Los niveles de ácido mirístico (C14:0) registraron valores de 2,56 y $2,55 \%$ en las líneas Criolla y Andina, reportándose diferencias estadísticas con respecto al contenido en carne de la línea Peruano mejorado, que obtuvo el $2,47 \%$ (Tabla I), esto sucede debido a las características específicas de cada línea; especies como conejos, ovinos y bovinos $(12,13,14)$ presentan niveles superiores, mientras que en suinos (15) los contenidos son inferiores a los de la carne de cuy.

En cuanto a los niveles de ácido pentadecanoico (C15:0) no se registraron diferencias estadísticas entre las líneas estudiadas, ya que se reportaron contenidos de 0,$73 ; 0,72$ y $0,71 \%$ en carne de cuyes Andino, Peruano mejorado y Criollo (Tabla I), valores superiores a los registrados en otras especies como conejos, ovinos y suinos $(12,13,15)$, pero inferiores al de los bovinos (14).

El contenido de ácido palmítico (C16:0) en la carne de cuy Criollo fue $27,81 \%$ (Tabla I), registrándose diferencias estadísticas con respecto a las líneas Andino y Peruano mejorado, que obtuvieron valores de 27,57 y $27,33 \%$, respectivamente; esto se debe a las características de cada línea; estos valores son similares a los reportados en conejos (12), inferiores a los registrados en ovinos, bovinos y aves $(13,14,16)$ y superiores a los de suinos (15).
Trabajos efectuados sobre el contenido de ácido heptadecanoico (C17:0) en carnes de conejos (12), ovinos (13) y suinos (15) reportan niveles inferiores, mientras que en bovinos (14), niveles superiores al de la carne de cuy; se registran valores de 0,95\% de este ácido en el Andino, identificándose diferencias estadísticas con relación a los datos obtenidos en el Peruano mejorado y el Criollo, que alcanzaron niveles de 0,91 y 0,89\%, respectivamente. (Figura I).

La Tabla I muestra que los contenidos de ácido esteárico (C18:0) en la carne de cuy Peruano mejorado, Andino y Criollo fueron 4,18; 4,15 y 4,08\%, respectivamente, no existiendo diferencias estadísticas entre ellos; estos valores son inferiores a los reportados en conejos (12), ovinos (13), bovinos (14) y aves (16), y superiores a los obtenidos en suinos (15).

La concentración de ácido araquídico en carne de cuy Peruano mejorado fue $0,53 \%$, registrándose diferencias estadísticas con relación a los valores obtenidos en la carne de cobayos Criollo y Andino, los cuales alcanzaron contenidos de 0,50 y $0,49 \%$ (Tabla I), existiendo variabilidad debido a las características propias de cada línea, reportándose concentraciones similares a las obtenidas en bovinos (14) e inferiores a las registradas en suinos (15).

Tabla I. Contenido de ácidos grasos saturados obtenidos de la carne de tres líneas de cuyes

\begin{tabular}{|l|l|c|c|}
\hline & \multicolumn{1}{|c|}{ Líneas de cuyes } & Media & Desviación típica \\
\hline \multirow{3}{*}{ Ácidos grasos saturados } & Peruano mejorado & $37,01^{\mathrm{a}}$ & 0,08 \\
& Andino & $36,71^{\mathrm{a}}$ & 0,09 \\
& Criollo & $37,11^{\mathrm{a}}$ & 0,05 \\
\hline \multirow{2}{*}{ Ácido láurico } & Peruano Mejorado & $0,57^{\mathrm{a}}$ & 0,01 \\
(C12:0) \% & Andino & $0,57^{\mathrm{a}}$ & 0,01 \\
\hline & Criollo & $0,56^{\mathrm{a}}$ & 0,01 \\
\hline Ácido mirístico & & $2,47^{\mathrm{b}}$ & 0,07 \\
(C14:0) \% & Peruano mejorado & $2,55^{\mathrm{a}}$ & 0,05 \\
& Andino & $2,56^{\mathrm{a}}$ & 0,02 \\
\hline
\end{tabular}




\begin{tabular}{|c|c|c|c|}
\hline & Líneas de cuyes & Media & Desviación típica \\
\hline Ácido pentadecanoico (C15:0) & $\begin{array}{l}\text { Peruano mejorado } \\
\text { Andino } \\
\text { Criollo }\end{array}$ & $\begin{array}{l}0,72^{\mathrm{a}} \\
0,73^{\mathrm{a}} \\
0,71^{\mathrm{a}}\end{array}$ & $\begin{array}{l}0.03 \\
0,05 \\
0,04\end{array}$ \\
\hline $\begin{array}{l}\text { Ácido palmítico } \\
(\mathrm{C} 16: 0) \%\end{array}$ & $\begin{array}{l}\text { Peruano mejorado } \\
\text { Andino } \\
\text { Criollo }\end{array}$ & $\begin{array}{l}27,33^{\mathrm{a}} \\
27,57^{\mathrm{b}} \\
27,81^{\mathrm{a}}\end{array}$ & $\begin{array}{l}0,10 \\
0,05 \\
0,19\end{array}$ \\
\hline $\begin{array}{l}\text { Ácido heptadecanoico (C17:0) } \\
\%\end{array}$ & $\begin{array}{l}\text { Peruano mejorado } \\
\text { Andino } \\
\text { Criollo }\end{array}$ & $\begin{array}{l}0,91^{\mathrm{b}} \\
0,95^{\mathrm{a}} \\
0,89^{\mathrm{c}}\end{array}$ & $\begin{array}{l}0,02 \\
0,02 \\
0,02\end{array}$ \\
\hline $\begin{array}{l}\text { Ácido esterárico } \\
\text { (C18:0) \% }\end{array}$ & $\begin{array}{l}\text { Peruano mejorado } \\
\text { Andino } \\
\text { Criollo }\end{array}$ & $\begin{array}{l}4,18^{\mathrm{a}} \\
4,15^{\mathrm{a}} \\
4,08^{\mathrm{a}}\end{array}$ & $\begin{array}{l}0,28 \\
0,39 \\
0,18\end{array}$ \\
\hline Ácido araquídico (C20:0) \% & $\begin{array}{l}\text { Peruano mejorado } \\
\text { Andino } \\
\text { Criollo }\end{array}$ & $\begin{array}{l}0,53^{\mathrm{a}} \\
0,49^{\mathrm{b}} \\
0,50^{\mathrm{ab}}\end{array}$ & $\begin{array}{l}0,04 \\
0,01 \\
0,01\end{array}$ \\
\hline
\end{tabular}

Letras diferentes sobre los promedios de caracterización físico-químicas indican diferencias significativas $(p \leq 0,05)$.

Como se observa en la Tabla II, los contenidos totales de ácidos grasos monoinsaturados en la carne de cuy no presentan diferencias estadísticas entre las tres líneas estudiadas, ya que registran valores de $30,49,29,26$ y $31,44 \%$, respectivamente, para las líneas Andino, Criollo y Peruano Mejorado; niveles superiores a los reportados en conejos (12) e inferiores a los registrados en aves (17).

Los niveles de ácido miristoleico (C14: 1cisn) en carne de cuy fueron de $0,21,0,20$ y $0,20 \%$ para las líneas de cuyes Andino, Criollo y Peruano Mejorado, no identificándose diferencias estadísticas (Tabla II); estos valores son superiores a los obtenidos en suinos (15) e inferiores a los registrados en ovinos (13); esto sucede debido a las características propias de cada especie.
Con relación al contenido de ácido palmitoleico (C16:1), la Tabla II indica que no hubo variabilidad entre las líneas de cuyes Andino, Criollo y Peruano Mejorado, no registrándose, por tanto, diferencias estadísticas, siendo estos niveles superiores a los reportados en conejos (12) e inferiores a los registrados en ovinos (13), bovinos (14), suinos (15) y aves (16).

El contenido de ácido elaidico (C18: 1transn9) en carne de cuy de la línea Peruano Mejorado fue de $0,78 \%$, reportándose diferencias estadísticas con respecto a los niveles obtenidos en las líneas Andino y Criollo, cuyos valores fueron de 0,67 y $0,64 \%$, respectivamente (Tabla II); contenidos inferiores a los reportados en estudios efectuados en ovinos (13), bovinos (14) y suinos (15): influyen en 
estas diferencias las características de la especie y la alimentación.

La Tabla II muestra que los niveles de ácido oleico (C18:1n9cis) fue de 28,55\% en la carne de la línea Peruano Mejorado, registrándose diferencias estadísticas con respecto a los niveles obtenidos tanto en la carne de las líneas de cuyes Andino y Criollo, que registraron 28,55 y $26,94 \%$, respectivamente, existiendo, por tanto, variabilidad de acuerdo con la línea; otras investigaciones demuestran que estos niveles son inferiores a los obtenidos en conejos (12), ovinos (13), bovinos (14) y suinos (15).

La Tabla II indica que los niveles de ácido erúcico en la carne de cuy de las líneas Andino, Criollo y Peruano Mejorado fueron de 0,30, 0,31 y 0,41\%, respectivamente, no registrándose diferencias estadísticas entre las tres; estos valores son superiores a los registrados en suinos (15).

Tabla II. Contenido de ácidos grasos monoinsaturados obtenidos de la carne de tres líneas de cuyes

\begin{tabular}{|c|c|c|c|}
\hline Análisis & Líneas de cuyes & Media & Desviación típica \\
\hline \multirow{3}{*}{ Monoinsaturados } & Andino & $30,49^{\mathrm{a}}$ & 0,16 \\
\hline & Criollo & $29,26^{\mathrm{a}}$ & 0,22 \\
\hline & Peruano mejorado & $31,44^{\mathrm{a}}$ & 0,24 \\
\hline \multirow{3}{*}{$\begin{array}{l}\text { Ácido Miristoleico } \\
\text { (C14: 1cisn ) \% }\end{array}$} & Andino & $0,21^{\mathrm{a}}$ & 0,01 \\
\hline & Criollo & $0,20^{\mathrm{a}}$ & 0,01 \\
\hline & Peruano mejorado & $0,20^{\mathrm{a}}$ & 0,00 \\
\hline \multirow{3}{*}{$\begin{array}{l}\text { Ácido Palmitoleico } \\
\text { (C 16:1) \% }\end{array}$} & Andino & $1,52^{\mathrm{a}}$ & 0,02 \\
\hline & Criollo & $1,51^{\mathrm{a}}$ & 0,02 \\
\hline & Peruano mejorado & $1,51^{\mathrm{a}}$ & 0,01 \\
\hline \multirow{3}{*}{$\begin{array}{l}\text { Ácido Elaidico } \\
\text { (C18: 1transn9) \% }\end{array}$} & Andino & $0,67^{\mathrm{b}}$ & 0,03 \\
\hline & Criollo & $0,64^{b}$ & 0,07 \\
\hline & Peruano mejorado & $0,78^{a}$ & 0,11 \\
\hline \multirow{3}{*}{$\begin{array}{l}\text { Ácido Oleico } \\
\text { (C18:1n9cis) \% }\end{array}$} & & $27,79^{a b}$ & 0,66 \\
\hline & Anaino & $26,94^{\mathrm{b}}$ & 0,30 \\
\hline & $\begin{array}{l}\text { Criollo } \\
\text { Peruano mejorado }\end{array}$ & $28,55^{\mathrm{a}}$ & 0,95 \\
\hline \multirow{3}{*}{ Äcido erúcico } & Andino & $0.30^{\mathrm{a}}$ & 0.04 \\
\hline & Criollo & $0,31^{\mathrm{a}}$ & 0,04 \\
\hline & Peruano mejorado & $0,41 \mathrm{a}$ & 0,04 \\
\hline
\end{tabular}

Letras diferentes sobre los promedios de caracterización físico-químicas indican diferencias significativas $(p \leq 0,05)$.

La Tabla III indica que el contenido total de ácidos grasos poliinsaturados en carne de cuy fue de
$13,30,11,04$ y $14,22 \%$, respectivamente, en las líneas Andino, Criollo y Peruano Mejorado; no registrándose diferencias estadísticas entre ellas; estos valores son inferiores a los reportados en conejos (12), ovinos (13) y aves (16), y superiores los registrados en bovinos (14). 
Tabla III. Contenido de ácidos grasos poliinsaturados obtenidos de la carne de tres líneas de cuyes

\begin{tabular}{|c|l|c|c|}
\hline \multicolumn{1}{|c|}{ Variable } & \multicolumn{1}{|c|}{ Líneas de cuyes } & Media & Desviación típica \\
\hline & Andino & $13,30^{\mathrm{a}}$ & 0,28 \\
Ácidos grasos poliinsaturados & Criollo & $14,04^{\mathrm{a}}$ & 0,24 \\
& Peruano mejorado & $14,22^{\mathrm{a}}$ & 0,25 \\
\hline
\end{tabular}

Letras diferentes sobre los promedios de caracterización físico-químicas indican diferencias significativas $(p \leq 0,05)$.

La Figura 1 indica que los niveles del ácido linoleico (C18:2n-6-omega 6) en la carne de las líneas Peruano Mejorado y Criollo fueron de 11,93 y $12,06 \%$, que difieren estadísticamente con respecto al valor encontrado en la carne de la línea Andino, que obtuvo un valor de $11,21 \%$; siendo estos niveles inferiores a los registrados en conejos (12), suinos (15) y aves (16), y superiores en ovinos (13) y bovinos (14).

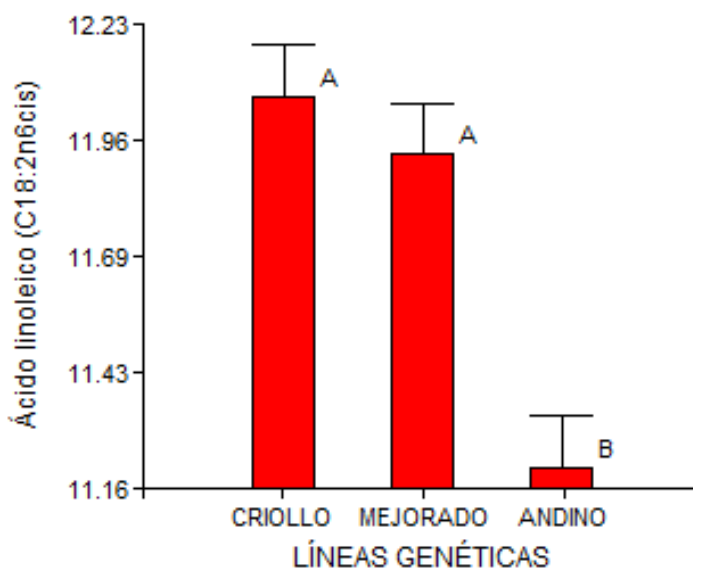

Figura 1. Contenido de ácido linoleico (C18:2n-6Omega 6) obtenido de la carne de tres líneas de cuyes

Los contenidos de ácido Alfa linolénico (C18:3n-3Omega 3) en la carne de cuy Peruano Mejorado fue de 2,29\%, estableciéndose diferencias estadísticas con respecto a los valores encontrados en las líneas Criollo y Andino, que fueron 1,98\% y $1,92 \%$, respectivamente (Figura 2 ); estos contenidos son superiores a los registrados en ovinos (12) y bovinos (13), e inferiores a los obtenidos en conejos (11) y aves (15).

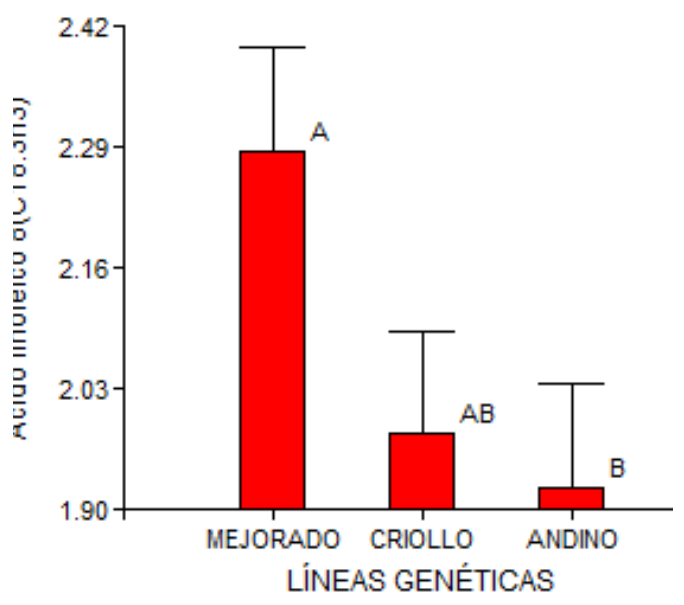

Figura 2. Contenido de ácido Alfa linolénico (C18:3n-3 - Omega 3), obtenido de la carne de tres líneas de cuyes

\section{Conclusiones}

El contenido total de ácidos grasos saturados no registró diferencias estadísticas entre las tres líneas estudiadas, ya que presentaron valores de $37,11 \%$, $37,01 \%$ y $36,71 \%$ en cuy Criollo, Andino y Peruano mejorado, respectivamente, siendo el ácido palmítico el más representativo en cuanto a su contenido.

Los contenidos de ácidos grasos monoinsaturados en la carne de cuy no presentan diferencias estadísticas entre las tres líneas estudiadas, ya que registran valores de $30,49 \%, 29,26 \%$ y $31,44 \%$, respectivamente, para las líneas Andino, Criollo y Peruano Mejorado, siendo el más representativo el 
ácido oleico (C18:1n9cis), ya que su contenido es más alto.

Los contenidos totales de ácidos grasos poliinsaturados en las líneas de cuyes Peruano mejorado, Criollo y Andino fueron 14,22\%, 14,04\% y $13,30 \%$, respectivamente, no registrándose diferencias estadísticas entre las líneas evaluadas, siendo el nivel más alto el del ácido linoleico (C18:2n6cis - omega 6).

\section{Referencias}

(1) Santos, V. El cuy y su importancia en el mercado. Rev. Arch. Latinoam. Prod. Anim. 2007; 15(1): 216-217.

(2) Chávez, S. Tecnologías de producción y comercialización de carne de cuy procesada para el mercado nacional y de exportación. Servicios educativos, promoción y ayuda rural. Junín, Perú. 2013.

(3) Argote, F.; Cuervo, R. Agroindustrialización de la carne de cuy. Revista Científica Guillermo de Ockham. 2012; 10(2): 217-218.

(4) RTE-INEN 056. Carne y productos cárnicos. Instituto Ecuatoriano de Normalización. 2013.

(5) Castañeda, R. y Peñuela, L. Ácidos grasos en carne. Sitio Argentino de Producción Animal. Córdoba, Argentina. 2010.

(6) Valenzuela, A. Ácidos grasos con isomería trans I, su origen y los efectos en la salud humana. Revista Chilena de Nutrición. 2012; 35(3): 162-171, 2008.

(7) Ballesteros, M.; Valenzuela, L.; Artalejo, E.; Robles, A. Ácidos grasos trans: un análisis del efecto de su consumo en la salud humana, regulación del contenido en alimentos y alternativas para disminuirlos. Revista Nutrición Hospitalaria. 2012; 27(1): 54-64.

(8) Argote, F.; Velasco, R.; Paz, P. Estudio de métodos y tiempos para la obtención de carne de cuy (Cavia porcellus) empacada al vacío. Revista de la Facultad de Ciencias Agropecuarias. 2007 ; 5(2): 103-111.
(9) ISO-15304. Animland vegetable fats and oils. Analysis by gas chromatography of methyl esters of fatty acids International Standarization Organization. 2012.

(10) Morales, J.; Quemé, J.; Melgar, M. Infostat, manual de usos. Centro Guatemalteco de Investigación y Capacitación. Guatemala. 2009.

(11) Castañeda, M.; Cabrera, A.; Navarro, Y. Procesamiento de datos y análisis estadísticos utilizando SPSS. Pontificia Universidad Católica de Río Grande de Sul. 2010.

(12) Calvache, I. Evaluación del contenido de ácidos grasos en la canal de conejos alimentados con morera (Morus alba). Universidad de La Salle. Bogotá, Colombia; 2005.

(13) Cruz, M.; Sánchez, D.; López, J.; Munguia, J.; Molina, R.; Rivera, F.; Hernández, J. Caracterización del perfil de ácidos grasos en carne de borrego de engorde utilizando cromatografía de gases. Revista NACAMEH. 2014; 8(1): 3949.

(14) Rincón, E.; Albarracín, W. Puesta a punto de un método analítico mediante cromatografía de gases para la determinación del perfil lipídico en carnes. VITAE, Revista de la Facultad de Química Farmacéutica. 2013; 20(2):111117.

(15) Prokopp, T.; Castro, A.; Hashime, E. Determination of the composition, cholesterol and fatty acid profile of cuts of meat trade ostrich, swine, beff and poultry. Brazilian Journal of Food and Nutrition. 2012; 23(2): 327-332.

(16) Salazar, I.; Rosado, J.; Chel, L.; Bentacur, D.; Castellanos, A. Composición en ácido graso alfa linolénico en huevo y carne de aves empleando chía. Revista Interciencia. 2009; 34(3): 209-213.

(17) González, D.; Civit, D.; Díaz, M. Composición de ácidos grasos en carne de corderos lechales y medianos de la raza Corridale. Revista Veterinaria Argentina. 2010; 27(267): 1-9. 